\title{
Identification of distinct upper and lower gastrointestinal symptom groupings in an urban population
}

\author{
N J Talley, P Boyce, M Jones
}

\begin{abstract}
Background-The current classification dividing patients with functional gastrointestinal symptoms into subgroups remains controversial.

Aims-To determine whether distinct symptom groupings exist in the community.

Methods-A random sample of Sydney residents in Penrith, Australia was mailed a validated self report questionnaire. Gastrointestinal symptoms including the Rome criteria for irritable bowel syndrome (IBS) and dyspepsia were measured.

Results-Among 730 respondents, the 12 month age and gender adjusted prevalence (adjusted to the Australian population) of IBS, dyspepsia, and gastro-oesophageal reflux were $11.8 \%(95 \%$ confidence interval (CI) 9.3 to $14.3 \%), 11.5 \%$ (95\% CI 9.6 to $14.6 \%$ ), and $17.5 \%$ (95\% CI 14.2 to $19.9 \%$ ), respectively. In total, $60 \%$ of the population reported four or more gastrointestinal symptoms. There was considerable overlap of IBS with dyspepsia and among the dyspepsia subgroups by application of the Rome criteria. Independently, 10 symptom groupings were identified by factor analysis. The underlying constructs measured by these factors were generally the major abdominal syndromes recognised by the Rome classification: dyspepsia, IBS, reflux, painless constipation, painless diarrhoea, and bloating, in addition to a number of more specific symptom groupings.

Conclusion-Gastrointestinal symptoms are common and overlap in the community, but distinct upper and lower abdominal symptom groupings can be identified. (Gut 1998;42:690-695)
\end{abstract}

Department of Medicine, University of Sydney, Australia N J Talley

M Jones

Department of Psychological

Medicine

P Boyce

Correspondence to: Dr N J Talley, Professor of Medicine, Department of Medicine, University of Sydney, Clinical Sciences Building, Nepean Hospital, Penrith, NSW,

Australia 2751.

Accepted for publication 18 December 1997
Keywords: functional bowel disease; irritable bowel syndrome; Rome criteria; dyspepsia; factor analysis

Much research interest has focused on the irritable bowel syndrome (IBS), which is characterised by abdominal pain and disturbed defecation, because this syndrome is very common in gastrointestinal practice and is costly. ${ }^{1-4}$ Thompson and Heaton in a landmark UK study of healthy volunteers reported that one fifth had functional bowel symptoms. ${ }^{5}$ Subsequently, a number of studies from Europe, the United States, and recently Australia have documented the prevalence of IBS in the community. ${ }^{6-13}$ However, the criteria used to document the presence of IBS have varied and most studies have not applied the now accepted Rome criteria for IBS. $^{2}$ Indeed, it remains unclear whether the variable prevalence rates reported are due to measurement error, as different measures (some not validated) or definitions have been applied, or because the prevalence truly varies from country to country or among ethnic groups. We have reported in a recent community based study, however, that the Rome criteria and Manning criteria identified a similar population. ${ }^{12}$

A key unresolved issue in the epidemiology of gastrointestinal symptoms is why there is a striking overlap of chronic upper and lower complaints..$^{1415}$ Indeed, a major Swedish population based study reported that IBS and dyspepsia could not be distinguished. ${ }^{10}$ The concept that different functional gastrointestinal disorders are the end result of a common set of pathophysiological disturbances (the "irritable gut") has been suggested as one explanation for the observed overlap. ${ }^{10}$ This concept is not established but has important diagnostic and therapeutic implications.

It remains controversial whether distinct subgroups of functional gastrointestinal disorder exist based on symptoms because of the paucity of empirical data on symptom groupings in the community; this represents a major deficiency in the literature. ${ }^{2}{ }^{10} 16$ One objective approach is to identify whether groupings of symptoms exist in the population that correlate with each other. Such a group of symptoms is analogous to a syndrome and the symptoms are more likely to be the result of a common underlying pathophysiology. ${ }^{10} 16$ We hypothesised a priori that there are several discrete groups of upper and lower gastrointestinal symptoms in the community.

We therefore aimed in this study to identify whether groups of symptoms occur together, based on application of factor analysis in a randomly selected population who completed a validated self report questionnaire.

\section{Methods}

ASSESSMENT OF SYMPTOMS

The Bowel Symptom Questionnaire (BSQ), derived from the Bowel Disease Questionnaire (BDQ) was applied in this study. ${ }^{17}{ }^{18}$ We have shown that the BSQ is easy to complete, highly reliable, and measures what it purports to measure (it is therefore valid) in the Australian community. The BSQ comprises 95 items currently including 48 symptom items. The symptom items were developed from the $\mathrm{BDQ}^{18}$ that has been validated and applied in population 
based studies in the United States and Germany. ${ }^{681415}$ The BSQ was not specifically designed to measure the Rome criteria symptoms but was developed to identify common upper and lower gastrointestinal complaints in the community. Additional questions were added after pilot testing on lifetime abdominal pain and bowel dysfunction.

\section{POPULATION SAMPLE}

In Australia, all adults (18 years and older) are required by law to be registered on the electoral rolls. We obtained the electoral roll for the district of Lindsay in the west of Sydney, Australia, as described elsewhere. ${ }^{12}{ }^{13}$ This district is primarily served by Nepean Hospital, a teaching hospital of the University of Sydney. This area, which includes Penrith, and surrounding suburbs, has a population of 73393 and is demographically very similar to the Australian population according to 1991 census data, except that it is slightly younger and has a slightly higher socioeconomic status. ${ }^{17}$

This study was approved by the local area Research and Ethics Committee. Randomly, subjects on the electoral roll were selected for the study $(n=1500)$. All subjects were then mailed an explanatory letter and the BSQ. Those who had moved from the area, had died, or were identified to have a serious illness that precluded them from taking part in the study were excluded. The list of selected subjects was divided into three batches of 500 subjects, and mailings of the BSQ to them were begun at intervals approximately two months apart, for ease of administration.

Subjects were offered a $\$ 2.00$ lottery ticket in return for completing the questionnaire. Subjects were given the option of refusing. Reminder letters were sent at two week intervals. At week 6 this included another questionnaire. At week 10, we attempted to telephone subjects who had not yet responded. Finally, the survey was closed at 12 weeks.

Of the 1500 people in the general community to whom we sent the questionnaire, 323 $(22 \%)$ had moved, and were excluded on that basis. Another $42(3 \%)$ were excluded due to serious illness, death, or language difficulties, leaving a potential sample receiving the questionnaire of 1135 subjects. By the close of the survey at the beginning of November 1995, 360 subjects ( $32 \%$ of the possible sample) had explicitly refused to participate and 45 had not replied.

Of the 730 respondents ( $64 \%$ response rate), the mean age was 45.2 (SD 14.6 , range 20-89) years. There was no difference between responders and non-responders with respect to socioeconomic status based on location of residences. Compared with the Australian population, respondents were slightly older $(69 \%$ at least 40 years compared with $61 \%$ in the population). The proportion of females in the responding sample was very similar to that of the Australian female population (53.7\% versus $51.4 \%$ ). Responders were also reasonably similar to the Australian population in terms of level of education and marital status as described in detail elsewhere. ${ }^{13}$
SYMPTOM GROUPS

A number of symptom groups were defined a priori as follows.

\section{Irritable bowel syndrome}

The Rome criteria, rather than the Manning criteria, were applied to identify IBS subjects. ${ }^{12} 1319$ IBS was defined as abdominal pain that kept recurring over a period of more than three months in the prior year, in combination with: (1) one or more of the following symptoms: (i) abdominal pain relieved by defecation, often (more than $25 \%$ of the time); (ii) more frequent and/or less frequent bowel movements with pain, often; or (iii) looser and/or harder stools with pain, often; and (2) two or more of the following: (i) more than three bowel movements a day, often and/or less than three bowel movements a week, often; (ii) stools very lumpy or hard, often and/or stools very loose or watery, often; (iii) incomplete rectal evacuation, often and/or urgency, often and/or straining, often; (iv) abdominal bloating or distension, often; or (v) mucus in stools.

\section{Dyspepsia}

This was identified using the Rome criteria $^{20}$ : recurrent or chronic pain or discomfort centred in the upper abdomen for three or more months. Those with only lower abdominal pain or discomfort were excluded from this grouping.

In addition, dyspepsia subgroups as recommended by the Rome committee were identified as follows ${ }^{20}$ :

Ulcer like - dyspepsia plus three or more of: well localised pain in the epigastrium; pain relieved by food often; pain relieved by antacids often; pain occurring before meals or when hungry often; night pain; and periodic pain.

Dysmotility like-dyspepsia plus three or more of: early satiety; postprandial fullness; nausea; retching and/or vomiting; bloating; and pain or discomfort aggravated by food.

Reflux like-dyspepsia plus heartburn and/or acid regurgitation once a week or more frequently.

Gastro-oesophageal reflux (symptomatic reflux) - this was defined as heartburn and/or acid regurgitation once a week or more frequently in those without dyspepsia. ${ }^{21}$ Less frequent reflux symptoms were not considered to avoid counting minor complaints.

\section{STATISTICAL ANALYSIS}

The 12 month prevalence rates (adjusted to the Australian population based on the age (10 year) and gender distribution from the 1991 census data) and 95\% confidence limits were estimated for individual symptoms.

To identify symptom groups based on subject responses to the BSQ, a factor analysis was undertaken as described below. The symptom items included in the factor analysis $(n=48)$ are all listed in table 1 . Of these, 39 were binary (typically present/absent) and nine were ordinal questions. Subjects responding "no" to abdominal pain in the prior year were scored as giving a negative response to all the subsequent pain questions. 
Table 1 Prevalence rates (\%) of symptoms in a random sample from Sydney, Australia

\begin{tabular}{|c|c|c|c|}
\hline Symptoms & $\begin{array}{l}\text { Woment } \\
(n=390)\end{array}$ & $\begin{array}{l}\text { Ment } \\
(n=336)\end{array}$ & $\begin{array}{l}\text { Overall } \neq \\
\left(n=726^{\star}\right)\end{array}$ \\
\hline \multicolumn{4}{|l|}{ Abdominal pain in prior year } \\
\hline Pain $>3$ months in the past year & 22.5 & 15.1 & 18.8 \\
\hline Pain severity (moderate or greater) & 29.6 & 21.2 & 25.3 \\
\hline Pain frequency (at least once a month) & 39.3 & 29.8 & 34.4 \\
\hline Upper abdominal pain & 12.8 & 11.5 & 12.1 \\
\hline Lower abdominal pain & 16.5 & 10.4 & 13.4 \\
\hline Both upper and lower abdominal pain & 9.9 & 6.5 & 8.1 \\
\hline Localised epigastric pain (one finger) & 17.2 & 11.6 & 14.3 \\
\hline Woken by pain from sleep at night & 18.4 & 12 & 15.1 \\
\hline Periodic pain & 25 & 16.5 & 20.1 \\
\hline Abdominal pain (any) & 39.5 & 29.7 & 34.5 \\
\hline Pain relieved by defecation often & 56.8 & 49.1 & 52.9 \\
\hline More bowel movements when pain begins often & 31.5 & 37 & 34.3 \\
\hline Less bowel movements when pain begins often & 27.8 & 15.8 & 21.7 \\
\hline Looser stools when pain begins often & 41.8 & 41.8 & 41.8 \\
\hline Harder stools when pain begins often & 22.6 & 21.6 & 22.1 \\
\hline Belching relieves pain often & 33.9 & 40.7 & 37.4 \\
\hline Antacid relieves pain often & 42 & 42.6 & 42.3 \\
\hline Food or milk relieves pain often & 19 & 22 & 20.5 \\
\hline Food aggravates pain often & 37.8 & 30.1 & 34.2 \\
\hline Pain before meals often & 16.3 & 19.2 & 17.8 \\
\hline Pain postprandially often & 50.5 & 52.2 & 51.3 \\
\hline \multicolumn{4}{|l|}{ Constipation in prior year } \\
\hline Less than 3 bowel move/week often & 21.1 & 6.3 & 13.5 \\
\hline Very lumpy stools often & 29.5 & 16.5 & 22.9 \\
\hline Feelings of incomplete evacuation often & 37.6 & 22.9 & 30.1 \\
\hline Straining often & 30.5 & 14.3 & 22.3 \\
\hline \multicolumn{4}{|l|}{ Diarrhoea in prior year } \\
\hline More than 3 stools/day often & 11.3 & 12 & 11.7 \\
\hline Very loose or watery stools often & 13 & 13.2 & 13.1 \\
\hline Urgency often & 34.6 & 27.8 & 31.1 \\
\hline Mucus in stools & 12.2 & 6.4 & 9.2 \\
\hline \multicolumn{4}{|l|}{ Upper gastrointestinal complaints in prior year } \\
\hline Early satiety often & 16.6 & 10.9 & 13.7 \\
\hline Food staying in stomach & 23.4 & 14.5 & 18.8 \\
\hline Nauseous & 25.2 & 13.6 & 19.3 \\
\hline Vomiting & 8.6 & 4.9 & 6.7 \\
\hline Retching & 11 & 8.3 & 9.6 \\
\hline \multicolumn{4}{|l|}{ Gastro-oesophageal reflux symptoms in prior year } \\
\hline Heartburn once a month or more & 23.2 & 22 & 22.6 \\
\hline Acid reflux once a month or more & 17.2 & 11.3 & 14.2 \\
\hline \multicolumn{4}{|l|}{ Other gastrointestinal symptoms in prior year } \\
\hline Abdominal bloating often & 44.4 & 18.8 & 31.4 \\
\hline Upper abdominal bloating often & 17.5 & 29.1 & 23.4 \\
\hline Lower abdominal bloating often & 40.8 & 36.7 & 38.7 \\
\hline Both upper and lower abdominal bloating often & 40.7 & 30.6 & 35.6 \\
\hline Visible distension often & 36.6 & 12.9 & 24.5 \\
\hline \multicolumn{4}{|l|}{ Lifetime symptoms $\mathbb{}$} \\
\hline Previous recurrent abdominal pain & 44.1 & 36.2 & 40.1 \\
\hline Previous pain for $>3$ months & 43.4 & 35.7 & 39.5 \\
\hline Onset of abdominal pain $>5$ years ago & 57.2 & 69.5 & 62.2 \\
\hline \multicolumn{4}{|l|}{ Previous pain location } \\
\hline Upper abdomen & 35.6 & 37.4 & 36.5 \\
\hline Lower abdomen & 44.4 & 34.7 & 39.5 \\
\hline Both upper and lower abdomen & 20.4 & 25.6 & 23.1 \\
\hline Previous bowel disturbance & 29.2 & 20 & 24.5 \\
\hline
\end{tabular}

^Four subjects provided incomplete data and so were excluded.

†Age (10 year age groups) and łage and gender adjusted for Australia (based on 1991 census data).

Often, $>25 \%$ of the time.

$\S$ Defined as occurring before the past 12 months.

The aim of the statistical analysis was to determine whether any underlying symptom groupings could be identified and related to predefined diagnostic groups. A factor analysis followed by varimax rotation was used because this approach yields factors which are statistically independent. ${ }^{22}$ The factor scores for each subject were computed using the regression method. This involved summing the product of factor loadings and item scores for each subject for each factor. The resulting variables have a unit normal distribution and are independent. In the analysis, every factor with an eigenvalue over 1.0 was used since we did not find that the first two to three factors explained the majority of the variance. For descriptive purposes, the pattern of items which loaded on a given factor was interpreted and a descriptive label applied. The extent to which the predefined diagnostic groupings (IBS, dyspepsia, mixed, and neither) were differentiated by symptom factors was assessed by plotting the profile of factor score means by symptom group.

\section{Results}

PREVALENCE OF INDIVIDUAL GASTROINTESTINAL SYMPTOMS

Table 1 shows the prevalence of symptoms in men and women, and the age and gender adjusted prevalence of symptoms overall. Among the total symptom items (counting all abdominal pain as one item), $91 \%$ of the sample reported one or more symptoms while $81 \%$ had two or more, $70 \%$ had three or more, and $60 \%$ had four or more symptoms.

PREVALENCE AND OVERLAP OF SYMPTOM GROUPS The 12 month age and gender adjusted prevalence of IBS was $11.8 \%$ (95\% confidence interval (CI) 9.3 to $14.3 \%$ ) using the Rome criteria.

A total of 92 subjects in the sample met the Rome criteria for dyspepsia for an age and gender adjusted prevalence of $12.1 \%$ (95\% CI 9.6 to $14.6 \%)$. Of these, $76(83 \%)$ could be classified as ulcer-like, dysmotility-like, or reflux-like dyspepsia and $16(17 \%)$ were not classifiable. While the membership of these subgroups varied, there was also substantial overlap of the classifiable patients: $27(35.5 \%)$ met both ulcer-like and dysmotility-like criteria, $28(36.8 \%)$ met both ulcer-like and refluxlike criteria, and $25(32.9 \%)$ met both dysmotility-like and reflux-like criteria.

Symptomatic gastro-oesophageal reflux was also common, being reported by 128 subjects for an age and gender adjusted prevalence of $17.0 \%$ (95\% CI 14.2 to $19.9 \%$ ). Frequent heartburn (at least once per week) was reported by $14.7 \%$ (95\% CI 12.0 to $17.4 \%$ ). Of those with IBS, $57 \%$ also had dyspepsia and $40 \%$ had frequent heartburn.

\section{FACTOR ANALYSIS}

Seven factors with eigenvalues greater than 1 and which appeared to describe broad underlying constructs were isolated following rotation and were readily interpretable as follows:

A dyspepsia grouping in the prior year characterised by upper abdominal pain.

An irritable bowel grouping in the prior year comprising lower abdominal pain and three of the Manning criteria (pain relief with defecation, looser and more frequent stools at pain onset).

A gastro-oesophageal reflux grouping in the prior year characterised by heartburn, acid regurgitation, nausea, and vomiting.

A chronic painless constipation grouping in the prior year characterised by infrequent lumpy stools, straining, and feelings of incomplete evacuation.

A chronic painless diarrhoea grouping in the prior year characterised by frequent loose stools and urgency.

A bloating grouping in the prior year characterised in addition by visible distension, postprandial fullness, and early satiety. 
Table 2 Factor loadings and eigenvalues in the factor analysis groupings

\begin{tabular}{|c|c|c|}
\hline Factor (item listed below factor) & Loading & Eigenvalue $/ \%$ variance \\
\hline Dyspepsia & & $14.0 / 31.2$ \\
\hline Upper abdominal pain & 0.8 & \\
\hline Abdominal pain & 0.6 & \\
\hline Relief with antacids & 0.7 & \\
\hline Epigastric pain & 0.7 & \\
\hline Relief with belching & 0.7 & \\
\hline Pain after meals & 0.7 & \\
\hline Pain severity & 0.6 & \\
\hline Pain frequency & 0.6 & \\
\hline Pain aggravated by food & 0.6 & \\
\hline Pain for more than 3 months & 0.6 & \\
\hline Periodic pain & 0.6 & \\
\hline Night pain & 0.5 & \\
\hline Reflux & & $2.3 / 5.2$ \\
\hline Heartburn & 0.4 & \\
\hline Acid regurgitation & 0.5 & \\
\hline Retching & 0.8 & \\
\hline Nausea & 0.7 & \\
\hline Vomiting & 0.8 & \\
\hline Irritable bowel & & $2.7 / 5.9$ \\
\hline Looser bowel movements with pain & 0.8 & \\
\hline More frequent bowel movements with pain & 0.8 & \\
\hline Pain relief with defecation & 0.7 & \\
\hline Lower abdominal pain & 0.7 & \\
\hline Chronic painless constipation & & $1.7 / 3.7$ \\
\hline Less than 3 stools/week & 0.7 & \\
\hline Straining & 0.8 & \\
\hline Lumpy or hard stools & 0.7 & \\
\hline Feelings of incomplete evacuation & 0.6 & \\
\hline Chronic painless diarrhoea & & $1.2 / 2.7$ \\
\hline More than 3 stools/day & 0.8 & \\
\hline Very loose, watery stools & 0.6 & \\
\hline Urgency & 0.5 & \\
\hline Bloating & & $2.4 / 5.3$ \\
\hline Abdominal bloating & 0.8 & \\
\hline Visible distension & 0.7 & \\
\hline Lower abdominal bloating & 0.7 & \\
\hline Upper abdominal bloating & 0.6 & \\
\hline Food stays in stomach & 0.6 & \\
\hline Early satiety & 0.4 & \\
\hline Lifetime functional bowel disorder & & $3.0 / 6.7$ \\
\hline Lifetime pain & 0.9 & \\
\hline Onset pain & 0.9 & \\
\hline Pain for 3 months or more & 0.7 & \\
\hline Bowel disturbance & 0.7 & \\
\hline Upper abdominal pain & 0.7 & \\
\hline Lower abdominal pain & 0.6 & \\
\hline Painful constipation & & $1.3 / 2.8$ \\
\hline Less bowel movements with pain & 0.8 & \\
\hline Harder bowel movements with pain & 0.8 & \\
\hline Rectal mucus & 0.6 & $1.1 / 2.5$ \\
\hline Meal related pain & & $1.1 / 2.5$ \\
\hline Pain relieved by food & 0.8 & \\
\hline Pain before meals & 0.6 & \\
\hline
\end{tabular}

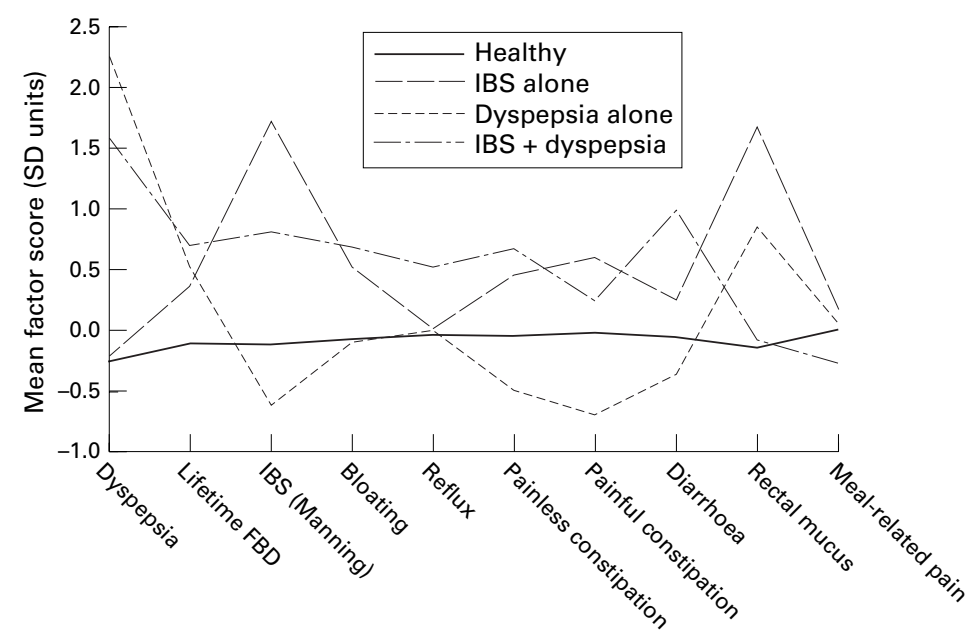

Latent variable

Figure 1 A comparison of the 10 symptom groupings derived from factor analysis and four a priori defined diagnostic groups based on the questionnaire responses (dyspepsia alone, irritable bowel syndrome alone, both dyspepsia and irritable bowel syndrome, and neither dyspepsia nor irritable bowel syndrome). Note the general similarity of the relevant factors across the diagnostic groups.
A functional bowel disorder grouping comprising pain and constipation or diarrhoea over the lifetime.

An additional three factors (painful constipation, mucus per rectum, and meal related pain) appeared to be individual items or pairs which did not correlate well with other items. Table 2 presents the factor loadings and eigenvalues.

Figure 1 summarises the extent to which the symptom profiles identified by the factor analysis overlapped or were distinct in the four generally accepted symptom groupings based on the Rome criteria, these being IBS alone, dyspepsia alone, both IBS and dyspepsia (mixed), and neither IBS nor dyspepsia (health). In doing so it provides an insight into the extent to which these symptom groupings are distinct entities. The 10 symptom groupings were as follows in fig 1 :

Factor 1: predominantly dyspepsia symptoms = dyspepsia;

Factor 2: lifetime functional bowel disorder $=$ lifetime FBD;

Factor 3: IBS symptoms approximating the Manning criteria = IBS (Manning);

Factor 4: symptoms indicating abdominal bloating = bloating;

Factor 5: predominantly reflux-like symptoms = reflux;

Factor 6: symptoms indicating chronic painless constipation $=$ painless constipation;

Factor 7: symptoms indicating painful constipation = painful constipation;

Factor 8: symptoms indicating chronic painless diarrhoea = diarrhoea;

Factor 9: a factor whose predominant item was the presence of rectal mucus $=$ rectal mucus;

Factor 10: symptoms indicating pain relieved by or preceding meals $=$ meal related pain.

The vertical axis in fig 1 presents the factor scores in standard deviation units. Mean scores of 2.0 or more are clearly elevated and suggest that the specified group is unlike the other groups. Mean scores between 1.0 and 2.0 are of interest. However, scores between 0.5 and 1.0 represent a grey area.

The healthy group provided a clear contrast with all other groups. This group exhibited low factor scores on all factors. The pure dyspepsia group was distinguished by a major elevation in dyspepsia symptoms and some elevation in rectal mucus, but low scores on IBS, constipation, and diarrhoea. The pure IBS group stood out as being notably high on the Manning criteria and rectal mucus, and somewhat elevated on chronic painful and painless constipation, as well as lifetime functional bowel disorder. The mixed dyspepsia/IBS group was, not surprisingly, distinguished by being a hybrid of the two pure groups. The mixed group showed some degree of elevation on most symptom factors, with the exception of rectal mucus. The mixed group did not however exhibit the extent of elevation on any particular symptom which the pure groups exhibited, with the exception of dyspepsia and diarrhoea. 
Table 3 Comparison of the 12 month period prevalence (\%) of irritable bowel syndrome in Australia, Germany and the United States using a similar validated questionnaire in random population based samples

\begin{tabular}{llcl}
\hline Country & Women & Men & Overall \\
\hline $\begin{array}{l}\text { Sydney, Australia } \\
\quad \text { urban) }\end{array}$ & $16(12-19)$ & $8(5-11)$ & $12(10-14)$ \\
$\begin{array}{l}\text { Essen, Germany† } \\
\quad \text { urban) }\end{array}$ & $16(11-21)$ & $8(5-13)$ & $12(9-15)$ \\
$\begin{array}{l}\text { Rochester, MN, USA } \\
\text { (semi-rural) }\end{array}$ & $14(10-17)$ & $12(9-15)$ & $13(11-15)$ \\
\hline
\end{tabular}

${ }^{\star}$ Rome criteria. Present study.

†Modified Rome criteria. Data from reference 14.

$\ddagger$ Manning criteria. Data from reference 6 .

\section{Discussion}

The present results confirm that upper and lower gastrointestinal symptoms are very common in the general population ${ }^{5-17}$ and provide some of the first comprehensive age and gender adjusted Australian population based data on individual symptoms. We applied a validated questionnaire to evaluate symptoms and the characteristics of the subjects responding appear to be representative of the sampling population frame. ${ }^{17}$ We have previously reported that the population sampled is similar to the Australian population sociodemographically. ${ }^{12} 1317$ While we recognise the need for caution in extrapolating our results, we believe that they are likely to be generalisable.

The prevalence of IBS varies by country but Kay and Jorgensen have suggested that studies applying different definitions of IBS are not comparable. ${ }^{23}$ We directly applied the standard Rome criteria in the present study and found that more than one in 10 persons in the community had a current diagnosis of irritable bowel syndrome (IBS). The findings in this population are consistent with studies from the United States and Germany that have applied a similar approach (table 3). In developing nations, much less data on the prevalence of IBS are available but Olubuyide et al recently reported that in apparently healthy medical students on a high fibre diet in Africa, the one year period prevalences of IBS using the Manning criteria in men and women were $24 \%$ and $48 \%$ respectively. ${ }^{24}$ Further work is needed to determine whether or not the prevalence of IBS differs in the developed compared with the developing world.

We did not undertake any medical evaluation in this study which may have resulted in an overestimation of the prevalence of functional bowel disorder. However, our results are comparable with other data ${ }^{5-11}$ and unrecognised structural disease is much less likely to contaminate a population based study. We therefore believe that the estimates are reasonably accurate.

We observed considerable overlap of IBS with dyspepsia and symptomatic gastrooesophageal reflux. Swedish investigators have reported that of persons with IBS, $87 \%$ also had dyspepsia. ${ }^{10}$ We found that of subjects with IBS in our study, $56 \%$ had dyspepsia. However, based on a factor analysis, natural groupings of upper and lower gastrointestinal symptoms could be identified in our sample. Our analysis suggests that gastrointestinal symptoms in the community fall into seven distinct groups. One of these groups comprised upper gastrointestinal symptoms consistent with dyspepsia while another was symptomatic gastrooesophageal reflux. Four consisted of lower gastrointestinal syndromes (IBS, chronic painless constipation, chronic painless diarrhoea, and lifetime functional bowel disorder). A distinct grouping had bloating with visible distension.

In this study we used factor analysis to isolate symptom groupings. It is therefore reassuring that the related groups were clinically meaningful, corresponding to recognised patterns of bowel dysfunction. Importantly, subjects suffering from specific clusters using the Rome criteria generally scored high on the appropriate symptom groupings and returned low scores on the other symptom groups (fig $1)$. It is of interest to note that in the "reflux" group, subjects with IBS and dyspepsia according to the Rome classification scored the highest. This may reflect the fact that gastrooesophageal reflux accounts for the presence of dyspeptic symptoms in IBS subjects. ${ }^{20}$

Other studies, while not population based, support our results. Whitehead et al factor analysed data on 23 bowel symptoms collected from two volunteer samples of women; they found that the factor accounting for most of the variance contained symptoms compatible with IBS but upper gastrointestinal symptoms were not evaluated. ${ }^{16}$ Similarly, Taub et al identified a similar IBS factor among 1344 psychology students surveyed at the University of Alabama. ${ }^{25}$ On the other hand, our results conflict with population based data from Agreus et $a l$ in Sweden; in their study, no distinct IBS and dyspepsia groupings could be identified using factor analysis. ${ }^{10}$ The Swedish investigators, however, used a different questionnaire that failed to measure all of the Rome or Manning criteria for IBS. Whether measurement bias, selectional factors, or true differences among populations account for the disparity remains to be determined.

The fact that the symptom groupings corresponded to the appropriate Rome criteria adds weight to the validity of the present syndrome definitions. It could be argued that the same criteria have been used to identify the symptom groups as well as the Rome criteria, in which case our findings would be circular. We would counter argue that this is not the case; the symptom groupings were identified from a "bottom up" data driven statistical approach compared with an approach where the subjects were independently classified using a priori predetermined criteria. Therefore, had the Rome criteria for IBS or dyspepsia been an arbitrary collection with no basis in reality, the data should have exhibited random profiles of factor scores across the four predefined groups. An additional proposition is suggested by the mixed IBS and dyspepsia cohort. The factor profile for this group exhibited elements in common with both the IBS and dyspepsia cohorts but also dissimilarities with both. This suggests the possibility that some aspects of both disorders may be modified by a dual disorder. Our current lack of knowledge about the 
temporal stability of the symptom groupings makes such conclusions speculative at this stage, and further work is needed. We believe that our findings are important because they provide confirmatory objective evidence that multiple functional gastrointestinal disorders do exist in the general population. However, the questionnaire we used was not specifically designed to measure all of the Rome criteria symptoms but rather to assess common upper and lower gastrointestinal tract symptoms. Whether further groupings of symptoms may be identifiable if more comprehensive surveys are applied needs to be determined. We also did not evaluate ethnic status and hence cannot determine whether our results apply to different ethnic groups, but others have not identified ethnic status to be a determinant of gastrointestinal symptom status. ${ }^{25}$

One distinct symptom grouping identified by the factor analysis related to lifetime symptoms. However, recall bias may have substantially affected the identification of these symptoms. Unfortunately, people have a well documented tendency to forget their gastrointestinal symptoms ${ }^{26}$ and even their fractured bones. ${ }^{27}$ Thus, prospective studies are needed to confirm the validity of this symptom grouping.

In conclusion, in an urban population based study we have identified a high prevalence of overlapping chronic gastrointestinal symptoms. In particular, we have shown that distinct groupings of symptoms exist in the community that are generally consistent with the Rome classification. While further confirmation of symptom groupings across different populations is now required, the data suggest that upper and lower functional gastrointestinal disorders are common but separate conditions in the general population.

Supported in part by a grant from the National Health and Medical Research Council of Australia.

1 Zighelboim J, Talley NJ. What are functional bowel disorders? Gastroenterology 1993;104:1196-201.

2 Drossman DA, Thompson WG, Talley NJ, et al. Identification of subgroups of functional gastrointestinal disorders. Gastroenterol Int 1990;3:159-72.

3 O'Keefe E, Talley NJ. Irritable bowel syndrome in the elderly. Clin Geriat Med 1991;7:265-86.

4 Talley NJ, Gabriel SE, Harmsen WS, et al. Medical costs in community subjects with irritable bowel syndrome. Gastroenterology 1995;109:1736-41.
5 Thompson WG, Heaton KW. Functional bowel disorders in 6 Talley NJ, Zinsmeister AR, Van Dyke C, et al. Epidemiology of colonic symptoms and the irritable bowel syndrome. Gastroenterology 1991;101:927-34.

7 Drossman DA, Li Z, Andruzzi E, et al. US householder survey of functional gastrointestinal disorders: prevalence, sociodemography and health impact. Dig Dis Sci 1993;38: 1569-80.

8 Talley NJ, Zinsmeister AR, Melton LJ III. Irritable bowel syndrome in a community: symptom subgroups, risk factors and health care utilization. Am $\mathscr{f}$ Epidemiol 1995;142:76-83.

9 Zuckerman MJ, Guerra LG, Drossman DA, et al. Healthcare-seeking behaviour related to bowel complaints. Dig Dis Sci 1996;41:77-82.

10 Agreus L, Svardsudd K, Nyren O, et al. Irritable bowel syndrome and dyspepsia in the general population: overlap and lack of stability over time. Gastroenterology 1995;109: 671-80.

11 Jones R, Lydeard S. Irritable bowel syndrome in the general population. BMF 1992;304:87-90.

12 Talley NJ, Boyce PM, Jones M. Predictors of health care seeking for irritable bowel syndrome: a population based study. Gut 1997;41:394-8.

13 Talley NJ, Boyce PM, Jones M. Is the association between irritable bowel syndrome and abuse explained by neuroticism? A population-based study. Gut 1998;42:47-53.

14 Holtmann G, Goebell H, Talley NJ. Dyspepsia in consulters and non-consulters. Prevalence, health care seeking behavour and risk factors. Eur $\mathcal{F}$ Gastroenterol Hepatol 1994;6: 917-24

15 Talley NJ, Zinsmeister AR, Schleck CD, et al. Dyspepsia and dyspepsia subgroups: a population-based study. Gastroenterology 1992;102:1259-68.

16 Whitehead WE, Crowell MD, Bosmajian L, et al. Existence of irritable bowel syndrome supported by factor analysis of symptoms in two community samples. Gastroenterology symptoms in two

17 Talley NJ, Boyce P, Owen BK, et al. Initial validation of a bowel symptom questionnaire and measurement of chronic gastrointestinal symptoms in Australians. Aust $N Z \mathcal{F}$ Med 1995;25:302-8.

18 Talley NJ, Phillips SF, Melton LJ III, et al. A patient questionnaire to identify bowel disease. Ann Intern Med 1989;111:671-4.

19 Talley NJ, Phillips SF, Melton LJ, et al. Diagnostic value of the Manning criteria in irritable bowel syndrome. Gut 1990;31:77-81.

20 Talley NJ, Colin-Jones D, Koch KL, et al. Functional dyspepsia: a classification with guidelines for diagnosis and dyspepsia: a classification with guidelines for diag

21 Locke GR III, Talley NJ, Fett S, et al. Prevalence and clinical spectrum of gastroesophageal reflux: a populationbased study in Olmsted County, Minnesota. Gastroenterology 1997;112:1448-56.

22 Armitage P, Berry G. Statistical methods in medical research. London: Blackwell, 1994:372-4.

23 Kay L, Jorgensen T. Redefining abdominal syndromes. Results of a population based study. Scand $\mathcal{F}$ Gastroenterol 1996;31:469-75.

24 Olubuyide IO, Olawuyi F, Fasanmade AA. A study of irritable bowel syndrome diagnosed by Manning criteria in an African population. Dig Dis Sci 1995;40:983-5.

25 Taub E, Cuevas JL, Cook EW, et al. Irritable bowel syndrome defined by factor analysis. Gender and race comparisons. Dig Dis Sci 1995;40:2647-55.

26 Heaton KW, Ghosh S, Braddon FE. How bad are the symptoms and bowel dysfunction of patients with the irritable bowel syndrome? A prospective, controlled study with emphasis on stool form. Gut 1991;32:73-9.

27 Jonsson B, Gardsell P, Johnell O, et al. Remembering fractures: fracture registration and proband recall in southern Sweden. F Epidemiol Community Health 1994;48:489- 